\title{
EMPLEO JUVENIL: ESCENARIO AL BICENTENARIO
}

\author{
YOUTH EMPLOYMENT: SCENE TO THE BICENTENNIAL \\ (iD) Ketty Marilú Moscoso Paucarchuco ${ }^{1 *}$, (iD) Manuel Michael Beraún Espíritu² \\ kmoscoso@unah.edu.pe; mberaun@continental.edu.pe \\ 1Universidad Nacional Autónoma de Huanta, Ayacucho, Perú \\ 2Universidad Continental, Junín, Perú \\ *Correspondencia: Ketty Marilú Moscoso Paucarchuco. Email: kmoscoso@unah.edu.pe
}

Recibido: 17.03.21 | Aprobado: 29.04.21

\section{RESUMEN}

El presente estudio es de carácter exploratorio descriptivo, con enfoque cualitativo, basado en una revisión documental, de tipo narrativa y tiene el objetivo de analizar la situación del empleo juvenil en el Perú, dentro del marco de las celebraciones del bicentenario por la independencia.

El desempleo ha golpeado duramente a la población peruana, más a los jóvenes y mucho más todavía a las mujeres jóvenes. Si bien es cierto los índices de su disminución muestran ligero favorecimiento, estos datos se verán modificados por la reciente situación pandémica por la que se está pasando. Es importante la intervención del Estado con políticas de protección y promoción del joven en la búsqueda de su primer empleo, brindándole solidez a través de la formación de habilidades duras y blandas, que le faciliten un desempeño competitivo en su labor. La empresa privada juega un rol básico y se refleja en su responsabilidad social empresarial; brindar las facilidades, incentivos y motivaciones a estas, es preocupación de los futuros líderes políticos de nuestro país.

Palabras clave: Empleo, empleo juvenil, desempleo, plan bicentenario.

\section{ABSTRACT}

This study is of a descriptive exploratory nature, with a qualitative approach, based on a documentary review, of a narrative type and has the objective of analyzing the situation of youth employment in Peru, within the framework of the celebrations of the bicentennial for independence.

Unemployment has hit the Peruvian population hard, more so on young people and even more so on young women. Although it is true that the rates of its decline show slight favoring, these data will be modified by the recent pandemic situation that is happening. The intervention of the State with policies for the protection and promotion of young people in the search for their first job is important, giving them solidity through the formation of hard and soft skills, which facilitate competitive performance in their work. The private company plays a basic role and is reflected in its corporate social responsibility; providing the facilities, incentives and motivations to these, is the concern of the future political leaders of our country.

Keywords: Employment, youth employment, unemployment, bicentennial plan.

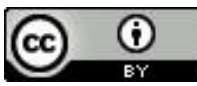




\section{INTRODUCCIÓN}

Las celebraciones por el Bicentenario de la Independencia del Perú, conlleva a un análisis profundo de la evolución sostenible del país, en líneas económicas, sociales y ambientales; en especial de aquellos que representan el futuro, nos referimos a los jóvenes y su situación de empleabilidad.

Las etapas del ser humano implican procesos de emancipación, desarrollo de proyectos de vida, traslado a la adultez que se vincula con la independencia. En estos escenarios el mercado laboral exige una alta competitividad, donde crece la oferta de servicios respecto a la demanda de mano de obra, a consecuencia de ello se genera una inestabilidad laboral que afecta a los jóvenes principalmente. La juventud implica inexperiencia, falta de contactos y desconocimiento, causales por la que muchos jóvenes quedan desempleados engrandeciendo la ausencia de capital social. Esto impide que la juventud se motive a ingresar a la "vida adulta", considerándola muy compleja y discriminatoria.

Para lograr un empleo decente, con emancipación, proyectos propios e integración social y que puedan enfrentar los obstáculos que implica la globalización y el crecimiento económico, es importante preparar a los jóvenes, adoptando políticas públicas de protección a la juventud, extendiendo redes de capacitación técnica profesional, que les ofrezca una seguridad social.

El empleo de los jóvenes se ha convertido en una preocupación importante en muchos países del mundo. Los responsables políticos dentro de sus discursos consideran prioridad ayudar a los jóvenes a realizar saludablemente la transición al mercado laboral y obtener un trabajo decente, pero las estadísticas manifiestan todo lo contrario. Es significativo analizar los programas que se han implementado para ayudar a los trabajadores jóvenes a encontrar trabajo; e identificar lo que parece funcionar en términos de mejora sobre los resultados de empleo para juventud.

De entrada, se debe reconocer que muchos jóvenes hacen la transición hacia su autonomía a través del empleo con éxito, sin necesidad de ninguna asistencia especial, por el contrario, otros experimentan dificultades, ya sea tardando mucho tiempo en establecerse o peor aún sin establecerse en el mercado laboral por completo. Ciertos grupos tienen problemas particulares, especialmente aquellos sin educación y sin habilidades básicas, quienes sufren más las consecuencias y se encuentran en una gran desventaja, son las mujeres jóvenes, jóvenes con discapacidad, minorías étnicas, migrantes, entre otros.

Tomar decisiones de cómo abordar el problema del desempleo juvenil en nuestro país, resulta relevante, tal y como se describe en el Plan Bicentenario a nivel nacional y en la Agenda 2030 de los Objetivos de Desarrollo Sostenible a nivel internacional; lamentablemente estas iniciativas de cara al bicentenario se han visto afectadas por el reciente problema pandémico, arrojando tasas altas en materia de desempleo, en especial en los jóvenes; pese al crecimiento económico pujante que tuvo nuestro país en los últimos tiempos. 
Esta diferencia social que marca el empleo decente en los jóvenes, genera un alto riesgo de exclusión social, el enfoque actual se concentra en la oferta de mano de obra, dejando de lado el fortalecimiento de estructuras económicas que den solidez a la demanda de trabajo. A fin de abordar el desempleo juvenil de manera más eficaz, es necesario generar nuevos empleos por políticas enfocadas de desarrollo económico (Salvia, 2013).

Parte de las funciones del Estado es la búsqueda del bienestar social, garantizar el acceso al mercado laboral con igualdad de oportunidades y equidad de género para mujeres, jóvenes, adultos mayores y personas discapacitadas principalmente (Díaz Llanos, 2020), por el contrario ha impuesto una legislación "flexibilizadora", impulsada especialmente en los años noventa, que acaba con buena parte de los derechos laborales y, con ello, la capacidad de presión por parte de las organizaciones sindicales (Adamovsky, 2012)

En este estudio se busca analizar la situación actual del empleo juvenil, y con ello sugerir propuestas de desarrollo y empoderamiento de nuestros jóvenes; para finalmente responder, ¿existe motivo de celebración por el bicentenario de independencia de nuestro Perú?

\section{EMPLEO}

Se entiende por empleo, a la actividad producida por una persona basada en esfuerzo físico y/o mental, que genera un valor para el empleador, el mismo que se retribuye con un salario o remuneración.

La población en edad de trabajar o población activa en el Perú y países de América Latina se calcula a partir de los 14 años. Para el año 2018, según resultados de la Encuesta Nacional de Hogares, la Población en Edad de Trabajar alcanzó las 24 millones 142 mil 300 personas, de las cuales, el 80,1\% residen en el Área Urbana y el 19,9\% restante en el Área Rural. Por otro lado, la Costa concentra el 57,6\% del total de la Población en Edad de Trabajar, la Sierra, el 30,2\% y la Selva, el 12,2\% (García Zanabria, José; Sánchez Aguilar, 2019); es decir que más de la mitad de la población activa se encuentra en la costa y reside en el área urbana del Perú.

Dentro de las estadísticas peruanas, respecto a la población en edad de trabajar, existe una disminución respecto al empleo decente en jóvenes de 14 a 24 años y un ligero incremento en adultos de 25 a 59 años; manteniéndose a lo largo de los años casi en el mismo porcentaje en personas mayores de 60 años. 


\section{Gráfico 1}

Población en edad de trabajar, según grupos de edad 2007-2019

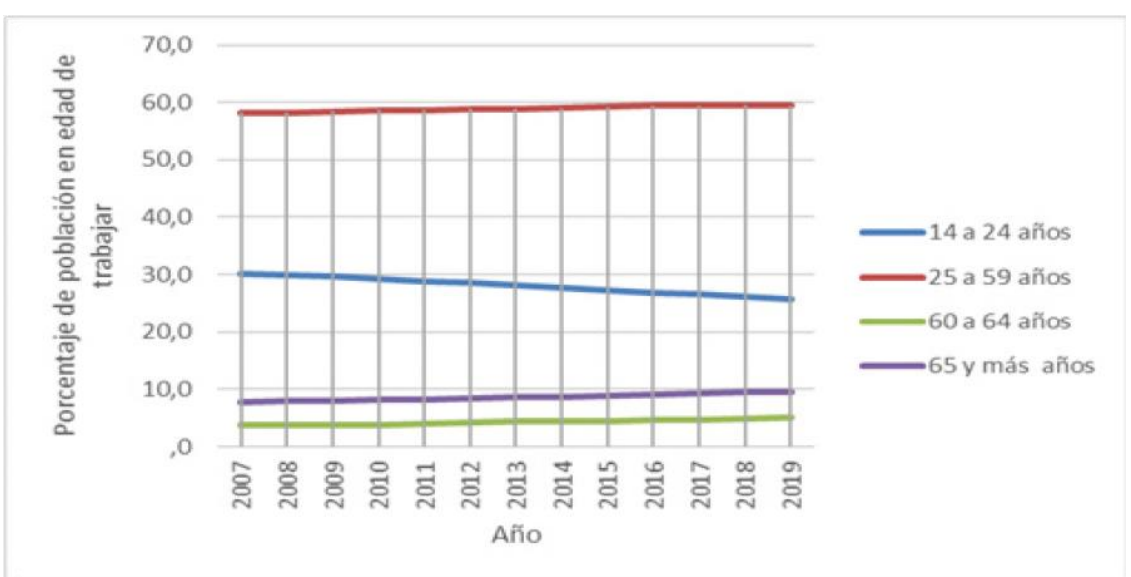

Fuente: Adaptado de (INEI, 2020)

El empleo en general no ha tenido una variación significativa, hasta antes de la pandemia cuyos índices han disminuido y no presentan todavía información de fuentes confiables, afectando más en el empleo juvenil.

\section{EMPLEO JUVENIL}

De acuerdo a la clasificación del Instituto Nacional de Estadística e Informática (INEI), así como, el Programa de las Naciones Unidas para el Desarrollo (PNUD), Organización Internacional del Trabajo (OIT), entre otras entidades, consideran población juvenil al grupo etario entre 15 a 29 años de edad (Galarreta Laurel, 2019). Revisando la línea de tiempo, se encuentran distintas políticas de empleo para jóvenes, en especial en el área de formación profesional y capacitaciones; así como la implementación de una serie de programas sociales, todo con la iniciativa de insertar en el mercado a los jóvenes y evitar el desempleo, subempleo, inactividad, contratos temporarios y/o autoempleo, muchas veces en el nivel de supervivencia (Galetto, 2016). Hasta antes de la pandemia el contexto mostraba una disminución de la desocupación de los jóvenes, quienes consiguen trabajos eventuales con facilidad y los pierden de la misma manera.

No está demás advertir el enorme déficit social acumulado durante años, a pesar de fortalezas económicas y que lamentablemente con un Plan Bicentenario difícilmente será resuelto. Más allá de este "difuso" panorama social, los costos que introducirá la pasada crisis se exhibirán en un aumento de la desocupación laboral, (mayoritariamente juvenil), la discriminación laboral femenina, una mayor vulnerabilidad social, una alta deserción escolar y la irrupción de la "trampa de la pobreza" (Quitral Rojas, 2012).

El problema del desempleo no se soluciona solamente con más años de educación (Pérez Aguilar, 2019), es importante analizar la situación y características actuales de la juventud, y generar programas de acuerdo a las necesidades de la población; evitando así que los jóvenes tengan la idea que para conseguir un trabajo decente tienen que sobre educarse a nivel de un estudio superior universitario, restando importancia a las carreras técnicas que en algunas circunstancias son las más demandadas. 
Los jóvenes tienen más probabilidades de estar desempleados o de estar contratados en trabajos precarios o de arreglo oscuro laboral, por lo tanto, carecen de una protección social adecuada. Al mismo tiempo, los jóvenes están respondiendo a la crisis a través de promoción de la salud, voluntariado e innovación. Las personas jóvenes constituirán un elemento clave en una recuperación inclusiva y el logro del Desarrollo Sostenible Objetivos (ODS) durante esta década de acción. Sin embargo, la respuesta y la recuperación deben realizarse de manera que proteja los derechos humanos de todos los jóvenes (Tarazona Leyva, Gloria Melina; Moscoso Paucarchuco, Ketty Marilú; Casas Cornelio, Cristian Jordy; Peña Chinguel, Reysenia; Quispe Santos, Claudia Adelayda; Sillo Campos, Anali Karin; Palomino Ramirez, 2021).

Los problemas de empleo juvenil tienen varias dimensiones y pueden manifestarse en diferentes formas. El más conocido es el desempleo; de hecho, las tasas de desempleo juvenil en general han aumentado durante la última década, al igual que la relación entre la tasa de desempleo de jóvenes y adultos. El desempleo juvenil es un problema que varía según la región; sin embargo, es una seria preocupación en todas partes.

A partir del 2007 al 2019, la población en edad de trabajar respecto al nivel de educación primaria ha disminuido de un $31,6 \%$ a un $25 \%$; respecto al nivel secundario, existe un ligero incremento del 41,4\% al 42,6\%; en el nivel superior no universitario, se genera un crecimiento del $13,1 \%$ al $15,1 \%$; y en el nivel superior universitario, se acrecienta de un $13,9 \%$ a un $17,3 \%$; concluyendo que la población en edad de trabajar respecto al año 2007, ha ido mejorando en su educación, lo que se refleja en los índices de crecimiento del empleo decente en términos generales.

Se puede manifestar que a nivel de la población en edad de trabajar hay un crecimiento significativo en educación superior universitaria y no universitaria, y hay una disminución pronunciada en la población activa de nivel de educación primaria.

\section{Gráfico 2}

Población en edad de trabajar, según nivel de educación, 2007-2019

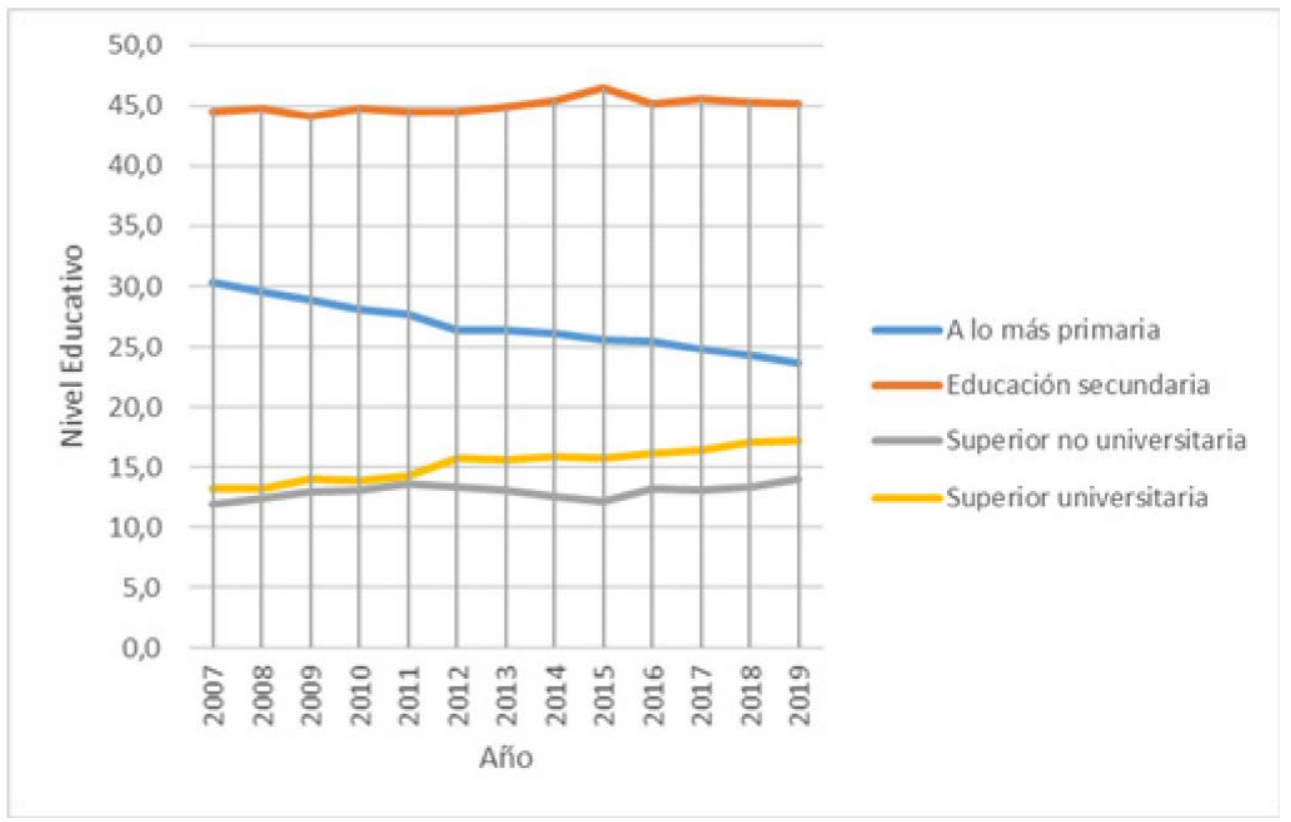


Fuente: Adaptado de (INEI, 2020)

Realizando este mismo análisis dentro la población activa del empleo juvenil, se haya un incremento importante en el fortalecimiento de la educación superior por parte de los jóvenes, que coincide con la situación latinoamericana.

\section{Gráfico 3}

Población ocupada de 14 a 29 años según nivel educativo, 2007-2017

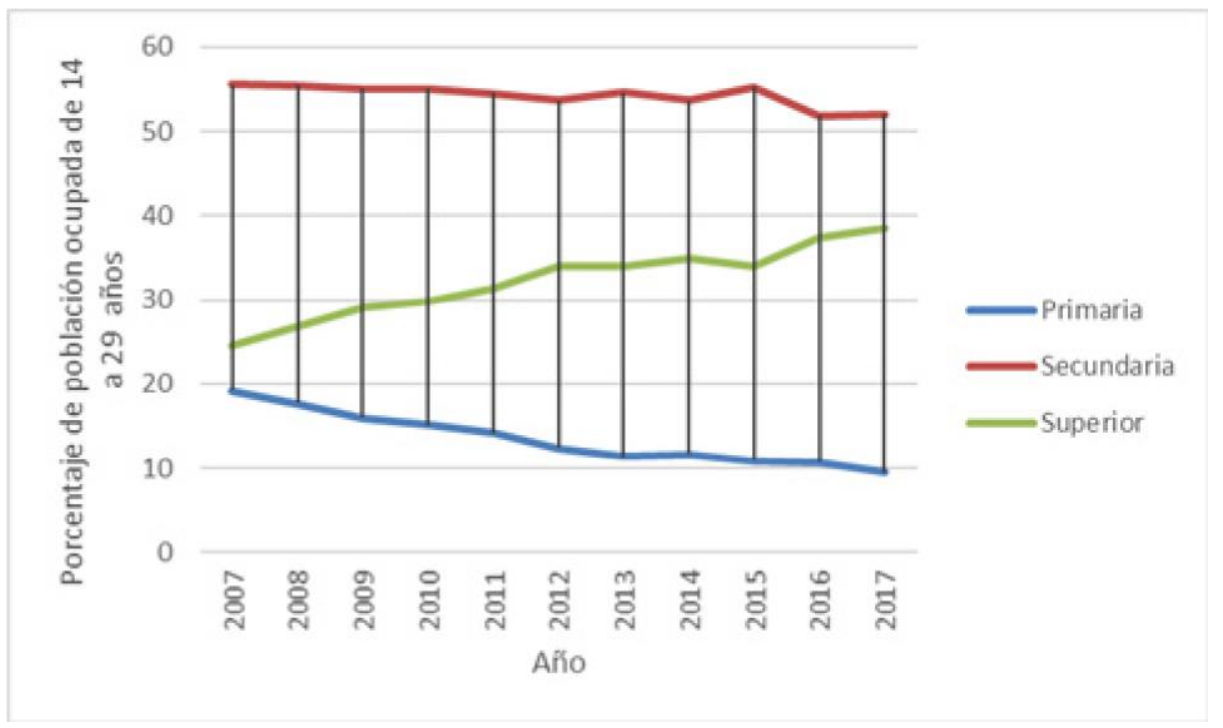

Fuente: Adaptado de (INEI, 2020)

Se puede manifestar que la juventud al no encontrar un empleo asalariado formal, inciden en trabajo poco remunerados e informales, o de lo contrario, se preocupa de su preparación académica para mejorar sus competencias, las mismas que son motivadas por su entorno familiar o amical. Para fortalecer ello, se crearon políticas de estado que lamentablemente no han funcionado, tal es el caso de la Ley de Régimen Laboral Juvenil, conocida como la Ley Pulpin, derogada en enero de 2015.

El desempleo juvenil ha generado un retraso de la asunción de los denominados "roles adultos", tales como la constitución de un hogar propio o de una familia, se ha masificado el estilo de vida "juvenil" y la vulnerabilidad laboral en los primeros años de inserción laboral (Miranda, Ana; Alfredo, 2018). Es importante desarrollar patrones de socialización, identidad y relaciones con otros pares a través de la familia, fortaleciendo el reconocimiento a nivel individual y colectivo, es allí en donde se forman los procesos de integración a la sociedad (Evans Morales, 2020), la misma que se refleja en un desarrollo regional y local; implantando ideas de industrialización para otorgar mayor empleo ciudadano, que mejore los niveles de ingreso per cápita.

\section{EL PLAN BICENTENARIO Y EL EMPLEO JUVENIL}

En el eje estratégico 4: Economía, competitividad y empleo, del Plan Bicentenario se sustenta una política económica estable y previsora que alienta el crecimiento económico sostenido mediante la inversión privada y pública en actividades generadoras de empleos dignos. De acuerdo con el Plan Bicentenario, son condiciones indispensables para este objetivo la reducción del subempleo y el desempleo, la mejora de la 
competitividad, la inversión y la presión tributaria, y la mayor estabilidad macroeconómica (CEPLAN, 2011).

Las diferencias sociales, pronunciadas por el poder adquisitivo, a causas de factores estructurales, es una problemática de múltiples matices (Olmos, 2011). Estas diferencias generan debilidad en el aspecto social e intercultural, precarizando las condiciones laborales preferentemente en los jóvenes pobres, reflejado en la explotación laboral, bajos ingresos salariales. Las distintas políticas adoptadas por el gobierno no logran atenuar las exorbitantes cifras de desempleo ya que distan del contexto actual, más todavía de este contexto pandémico, determinando que, bajo estas circunstancias, no hay motivo de celebración nacional por el Bicentenario.

El eje productivo del Plan Bicentenario, evalúa las opciones para el aumento del ingreso y de la demanda de empleo local teniendo en cuenta el mejoramiento de las empresas existentes, las empresas recuperadas y el impulso y creación de emprendimientos socioproductivos y aquellos de base tecnológica (Battista, Susana; Fusca, 2019). A lo largo de los años se ha observado la explotación de nuestro capital natural, reflejado en un consumismo extremo; que lamentablemente no ha ofrecido una propuesta de valor tan significativa como los productos de importación. Queda seguir trabajando en el potencial humano, que permita una economía circular, con aprovechamiento máximo de los recursos; esto generará mayor posibilidad de empleo decente.

Los programas de capacitación y empleo, buscan fortalecer el capital humano, a través de una preparación académica y experiencia que se otorgan mediante colectivos como: grupos de base, alianzas con empresa públicas y privadas, planes de desarrollo prospectivo en movimientos juveniles, y por supuesto acompañamiento y asesoría. A pesar de estos esfuerzos, se observa discriminación a jóvenes en especial mujeres de bajo nivel educativo, otro factor es el nivel socioeconómico del que provienen los jóvenes ya que, aunque puedan tener similar educación los miembros de los quintiles más bajos con los más altos, no presentan el mismo nivel de capital social o económico que les permita tener el apoyo necesario para acceder a un trabajo digno (Muñoz Martinez, 2018). Al margen de cualquier herramienta de gestión como el Plan Bicentenario, todos los esfuerzos deben volcarse en un desarrollo integral del joven, que no solo abarca habilidades duras (conocimientos), si no también habilidades blandas (sentimientos, carácter).

\section{SITUACIÓN ACTUAL DEL DESEMPLEO JUVENIL}

En el Perú, la problemática de desempleo juvenil, ha sido un tema que las políticas nacionales siempre han enfocado al sector privado, como actores para solucionarlo, claro está como parte de su responsabilidad social; el Estado ha cumplido un rol director y promotor, pero no ha cumplido el rol protagónico como debiera corresponder, siendo ellos el camino a una oportunidad para los jóvenes tengan un trabajo digno y decente (Pérez Espíritu, 2020). Actualmente las convocatorias de trabajo en el sector público, apertura poca, por no decir, nula posibilidad a un profesional que no cuenta con experiencia, los perfiles para los puestos de asistente, por ejemplo, exigen años de experiencia, dejando sin oportunidad al joven que solicita su primer empleo, por lo que, 
no hay políticas que ayuden al cumplimiento de la responsabilidad social empresarial estatal.

Las estadísticas muestran un leve crecimiento del empleo adecuado en los jóvenes (14 a 24 años) del año 2016 al 2017; del 32, 3\% a 33,2\%; la tasa de subempleo disminuye de $56,9 \%$ a $56,1 \%$ y la tasa de desempleo de $10,8 \%$ a $10,7 \%$. Tal y como se refleja en el siguiente gráfico.

\section{Gráfico 4}

Tasa de empleo adecuado, subempleo y desempleo, según grupos de edad. 2016-2017

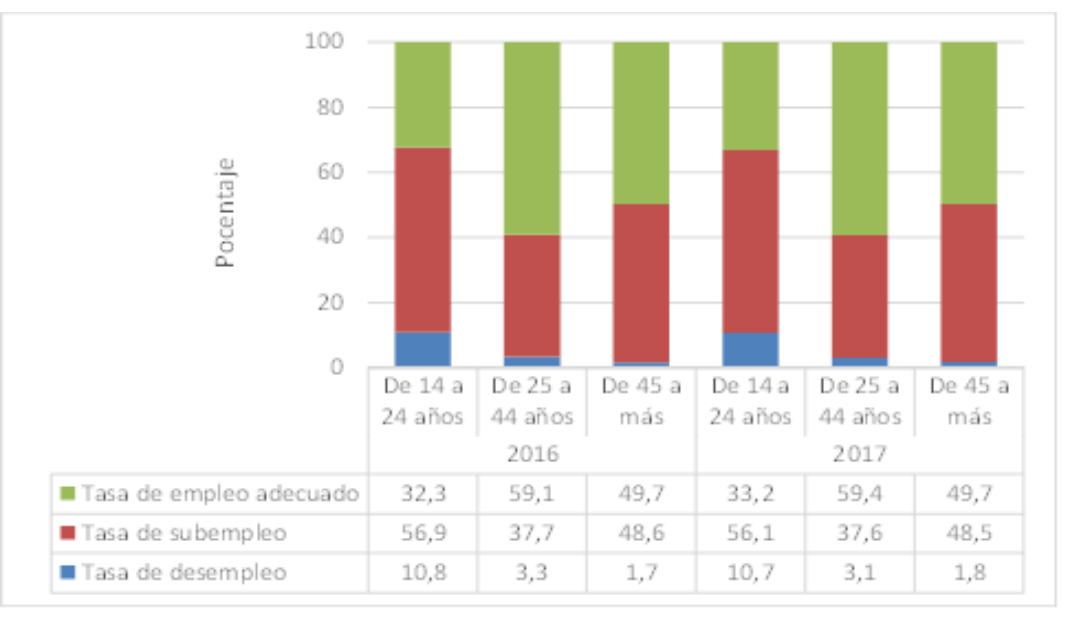

Fuente: Adaptado de (INEI, 2018)

La población ocupada joven (14 a 29 años), disminuye el empleo informal de un $88,4 \%$ a $79,1 \%$, creciendo así el empleo formal de un $11,6 \%$ a un $20,6 \%$, datos que sufrirán cambios severos a causa de la pandemia que ha mostrado las más grandes deficiencia en empleo a causa de la informalidad.

\section{Gráfico 5}

Población ocupada joven de 14 a 29 años por empleo formal e informal 2007-2016-2017

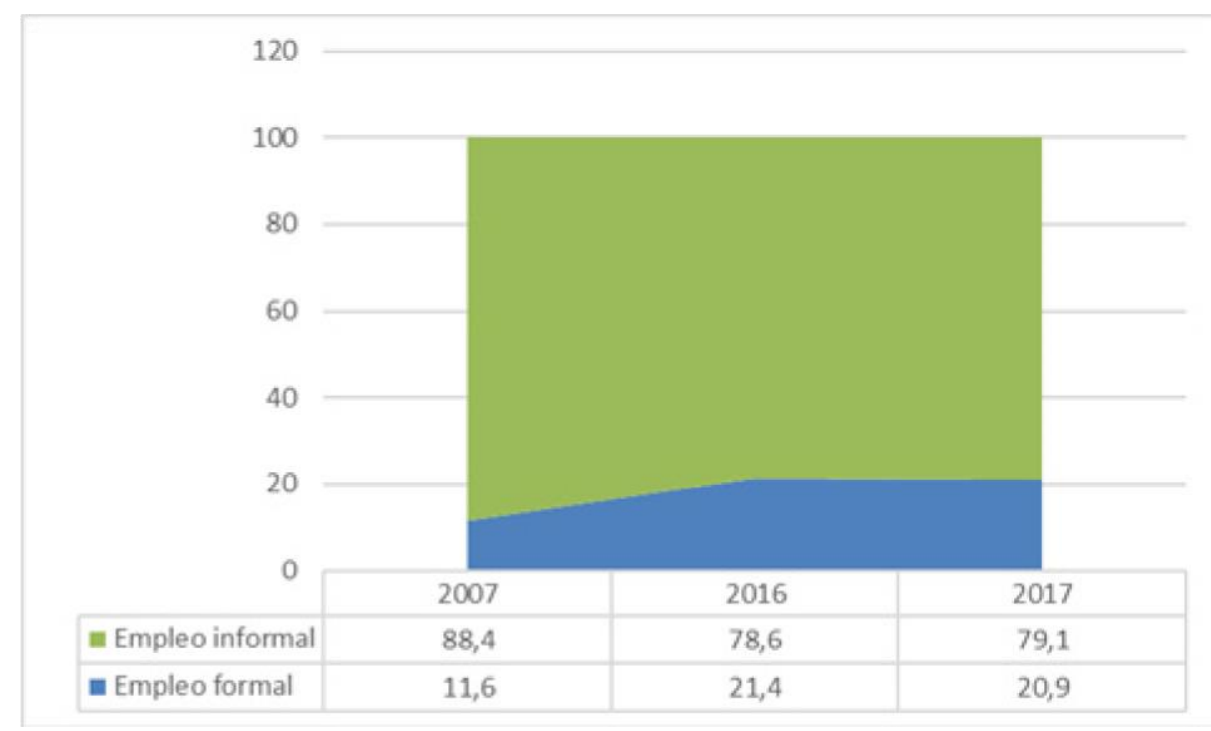

Fuente: Adaptado de (INEI, 2018) 
Respecto a los jóvenes desempleados según sexo, se observa una disminución desde el año 2007 al 2017, con casi 4 puntos porcentuales en los varones y 2,12\% en las mujeres.

\section{Gráfico 6}

Población desempleada y joven de 14 a 29 años según sexo 2007-2017

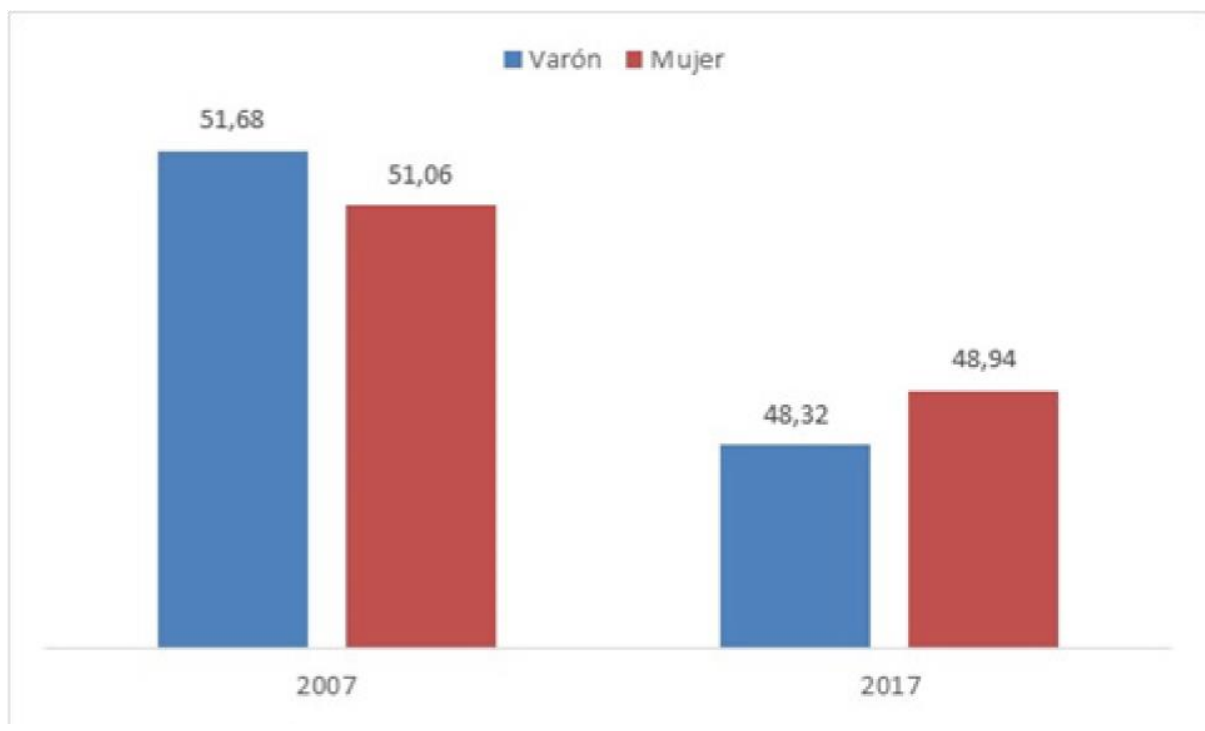

Fuente: Adaptado de (INEI, 2018)

En resumen, la situación de desempleo en los jóvenes a lo largo de estos últimos años ha sido muy flexible, siendo las mujeres, quienes cuentan con tasas mas altas de desempleo respecto a los varones, también es bueno recalcar que hay una disminución de este problema social.

\section{Gráfico 7}

Tasa de desempleo de la población de 14 a 29 años según sexo 2007-2017

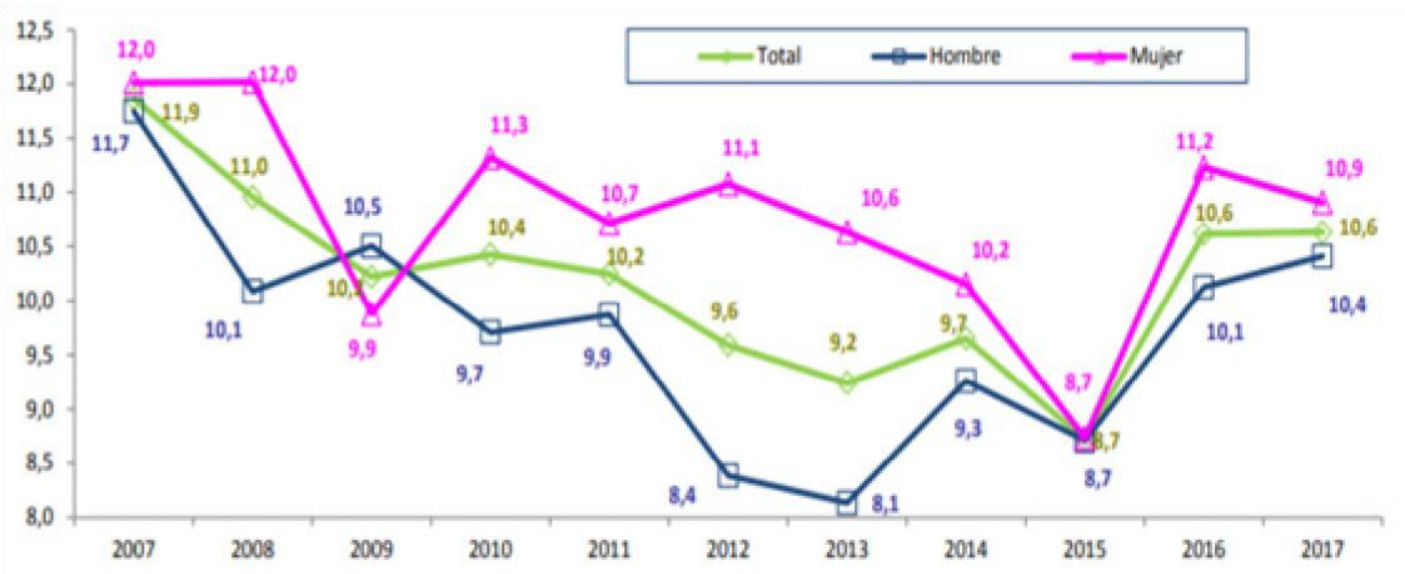

Fuente: Adaptado de (INEI, 2018)

El desempleo y el subempleo entre los jóvenes conlleva a un elevado costo socioeconómico lo cual representa una amenaza en el desarrollo de los países, en nuestro país todavía no se ha logrado implementar una política pública capaz de generar empleo decente para este grupo de la población (Cabrera Troya, 2016). Pese al discurso de la equidad de género, todavía no se ha podido erradicar la discriminación a las mujeres en el 
trabajo, ya que sigue siendo subestimada al asumir un cargo de alto nivel en una organización y su remuneración de condiciona a su género; felizmente existen variedad de casos donde las valoran por su formación y tareas asignadas.

\section{PROPUESTAS DE DESARROLLO}

Pese a la problemática económica y social, la intervención de los entes públicos y privados, facilita la "transición" de los jóvenes hacia su independencia que se vincula a su primer trabajo, por ello es elemental considerar a los grupos de interés para que sienten bases para la creación y fortalecimiento de carreras profesionales universitarias y técnicas, competitivas y de alta demanda.

Es primordial examinar las áreas clave de políticas para ampliar oportunidades de empleo para los jóvenes, mas ahora en tiempos de pandemia, dado que los jóvenes sufren de manera desproporcionada por la debilidad del trabajo demandada; la salud general de la economía es fundamental, esto subraya la importancia de buenas condiciones macroeconómicas y un clima de inversión positivo. Sin estas condiciones previas, los jóvenes tendrán escasas oportunidades de empleo. Por supuesto, la inversión en capital humano a través de la educación formal y de calidad es fundamental para aprovechar estas oportunidades. Las políticas del mercado laboral, instituciones y programas pueden marcar una diferencia significativa en términos de crear oportunidades para los jóvenes, mejorando sus planteamientos en pro de los que más necesitan.

En la Agenda 2030 se busca lograr el empleo pleno y productivo y el trabajo decente para todas las mujeres y los hombres, incluidos los jóvenes y las personas con discapacidad, así como la igualdad de remuneración por trabajo de igual valor. De acuerdo a la evaluación del plan al 2020, se debió reducir considerablemente la proporción de jóvenes que no están empleados y no cursan estudios ni reciben capacitación, desarrollar y poner en marcha una estrategia mundial para el empleo de los jóvenes y aplicar el Pacto Mundial para el Empleo de la Organización Internacional del Trabajo (Naciones Unidas/CEPAL, 2019), lo que se encuentra lejano de nuestra realidad. Las soluciones a nivel macroeconómico fortalecen las oportunidades de nuestros jóvenes, la evaluación del rendimiento de estos pactos, nos permitirá determinar la efectividad del apoyo internacional, de ser el caso aprovecharla al máximo, de lo contrario modificar estrategias y fortalecer las políticas nacionales resultan una gran responsabilidad de nuestras autoridades.

Es importante reconocer las dinámicas juveniles en los diferentes contextos, generar procesos de participación sostenibles, donde no se cree dependencia al profesional, crear redes de apoyo para fomentar el intercambio de saberes y conocimientos, la intervención y los proyectos sociales se deben realizar de forma articulada con las diferentes instituciones, teniendo en cuenta la voz de las comunidades juveniles (Buelvas Narváez, 2018). Los organismos existentes parecen insuficientes, se podrían crear nuevos organismos de planificación y de coordinación que deberían implicar más las organizaciones empresariales y sindicales. Organismos nacionales e internacionales deberían reabrir el debate sobre los diagnósticos teóricos del desempleo juvenil y sus implicaciones políticas (Salvia, 2013). 
Es importante resaltar la educación y la capacitación para la movilidad ascendente y el desarrollo integral de las personas, el contar con una perspectiva de futuro y de justicia intergeneracional; de la participación democrática, de la familia y la dignidad de las personas (García Delgado, 2010). Son estos principios asociados a la moralidad, las que impulsan a la sociedad a un compromiso de protección a quienes consideramos el "futuro de nuestro país", por ello es importante generar planes inclusivos dirigidos a jóvenes en situación de pobreza y pobreza extrema. Estas decisiones no pueden estar ajenas al pensamiento juvenil, se tiene que crear ambientes de diálogo y debate para los nuevos ciudadanos.

El apoyo al emprendimiento productivo e intermediación laboral en un diseño de orden nacional capaz de recoger las particularidades territoriales a través de un enfoque de desarrollo económico y local con participación del actor productivo-empresarial (Thezá, 2010). La primera participación en el mercado laboral del joven, marca los lineamientos de su éxito personal, por eso es importante acompañarlo en este riesgo a través de un fondo de capital que le permita desarrollar sus ideas de emprendimiento, como el presupuesto no es del todo suficiente, es importante también generar programas compatibles con su nuevo trabajo.

El rol de los empleadores es vital; pero muchos de ellos no invierten en la formación de sus trabajadores, menos aún si son jóvenes. Concientizar a los empresarios respecto a su responsabilidad social empresarial coadyuvaría a una formación académica, tecnológica y de habilidades en pro de la competitividad comercial de su organización, así como la mejora de la calidad de sus bienes o servicios. La motivación desde el Estado, debe verse reflejado en ciertos beneficios que compensen el cumplimiento de la labor social en contra del desempleo juvenil.

Estas propuestas de desarrollo deben apoyarse en una información sólida de acceso homogéneo a todos los jóvenes, sometiéndose a evaluaciones transparentes, que no se vean manchados por actos de corrupción, común en estos tiempos.

\section{CONCLUSIONES}

- Se entiende por empleo juvenil a aquella actividad física o intelectual realizada por jóvenes entre 14 y 29 años, cuya contraprestación es un salario de carácter dinerario generalmente.

- Los problemas del empleo juvenil surgen a causa de la inestabilidad económica a nivel macro; y de las debilidades en el soporte educativo, económico y social principalmente a nivel micro.

- Referido al empleo juvenil, no hay motivos de celebración por el Bicentenario de la Independencia, la evaluación de esta herramienta de gestión, traducido en un plan, muestra un ligero crecimiento, así como las diferencias de empleo entre varón y mujer a lo largo de estos años. Sobresaliendo grandemente el subempleo, seguido del empleo adecuado y el desempleo.

- La informalidad es uno de los problemas más pronunciados que impiden el crecimiento de un trabajo decente, llegando a un 79,8\% en el año 2017, con una proyección mucho mayor en el año 2021, a causa de la pandemia. 
- El Estado juega un rol fundamental, debido al diseño de sus políticas de empleo juvenil, aportar propuestas acordes a nuestra realidad o contexto, es su responsabilidad. Estas tienen que tener un sentido de protección y promoción de este grupo vulnerable; de la mano de los entes privados, para generar enfoques integrales que superen este mal social denominado desempleo juvenil.

- Formar a nuestros jóvenes con habilidades duras y blandas, genera competitividad y sostenibilidad empresarial, todo dentro de ambientes de dialogo y debate de estos grupos de interés, basados en información y transparencia, lejos de la corrupción.

- Consecuentemente, el análisis de la situación de desempleo de estos individuos, es a causa de la pobreza, problema que no se soluciona solo con plasmar letras en una herramienta de gestión como lo es el Plan Bicentenario, cuyo objetivo no se ha cumplido, existe desempleo juvenil y esta discriminación se inclina más hacia las mujeres.

- La juventud de estos tiempos es muy dinámica y cambiante, lo vimos reflejado en nuestros "jóvenes bicentenarios", quienes a gritos pidieron reforma política. Es en este marco de celebración donde se necesitan respuestas concretas a esta problemática social y de envergadura; más aún en estos espacios de cambio de gobierno, donde surgen promesas que buscan el "desarrollo" de nuestro querido Perú.

\section{REFERENCIAS BIBLIOGRÁFICAS}

Adamovsky, E. (2012). Conflictos y negociaciones por la definición de un ethnos nacional, de la crisis al Bicentenario. In G. J. für G. Lateinamerikas (Ed.), El color de la nación argentina (pp. 343-364). https://ri.conicet.gov.ar/bitstream/handle/11336/ 26581/CONICET_Digital_Nro.19668_A.pdf?sequence=4\&isAllowed=y

Battista, Susana; Fusca, N. (2019). El Estado como Conductor en el proceso de construcción de Políticas Públicas. Journal of Chemical Information and Modeling, 53(9), 1689-1699. http://biblioteca.municipios.unq.edu.ar/modules/mislibros/ archivos/FuscaBattistaRedMuni2010.pdf

Buelvas Narváez, Y. M. (2018). SISTEMATIZACIÓN DE LA EXPERIENCIA EN EL PROCESO DE EMPODERAMIENTO JUVENIL, IMPLEMENTADO POR LA FUNDACIÓN MARIO SANTO DOMINGO EN LA COMUNIDAD CIUDAD DEL BICENTENARIO [Universidad de Cartagena]. In Universidad de Cartagena (Vol. 2). https://repositorio.unicartagena.edu.co/bitstream/handle/ 11227/7289/Yolibeth Buelvas - Sistematizacion de experiencias 2018.pdf? sequence $=1$

Cabrera Troya, M. J. (2016). Los jóvenes como un grupo vulnerable dentro del mercado laboral y análisis del Proyecto Mi Primer Empleo, como componente de la política pública para el empleo juvenil en el Ecuador, en el periodo 2007-2013 (p. 84). http://repositorio.puce.edu.ec/bitstream/handle/22000/11485/DISERTACION EMPLEO JOVEN_MJoseCabrera.pdf?sequence=1\&isAllowed $=\mathrm{y}$

CEPLAN. (2011). PLAN BICENTENARIO El Perú hacia el 2021 (Primera). http:// library1.nida.ac.th/termpaper6/sd/2554/19755.pdf 
Díaz Llanos, M. del P. (2020). Propuesta de medidas jurídicas para la reinserción y permanencia laboral del adulto mayor en las políticas públicas peruanas. IUS Revista de Investigación de La Facultat de Derecho, 9, 18-39. https://doi.org/https:// doi.org/10.35383/ius-usat.v9i2.449

Evans Morales, F. (2020). Jóvenes, participación y espacios de poder en organizaciones de Lima Metropolitana. Aproximaciones rumbo al Bicentenario. Revista de Sociología, 31, 161-184. https://doi.org/10.15381/rsoc.v0i31.19280

Galarreta Laurel, L. R. (2019). EMPLEO JUVENIL Análisis de los factores que influyen en la inserción laboral. Caso: Usuarios del Programa Nacional de Empleo Juvenil “Jóvenes Productivos" del distrito de Ate en Lima Metropolitana durante el 2016 [Pontificia Universidad Católica del Perú]. https://doi.org/10.30875/bf5bbffe-es

Galetto, S. (2016). DOSSIER: FORO UNIVERSITARIO DEL BICENTENARIO ¿Cómo pensar la Política social para jóvenes que ingresan al mercado de trabajo en Argentina en el marco del bicentenario? KAIROS. Revista de Temas Sociales, 20(38). http:/ / www.revistakairos.org/wp-content/uploads/Galetto.pdf

García Delgado, D. (2010). Crisis global, modelos de desarrollo y Bicentenario. Interrogantes sobre el «bien común». Estudios Sociales, 39(1), 47-72. https://doi.org/10.14409/es.v39i1.2664

García Zanabria, José; Sánchez Aguilar, A. (2019). Perú: Evolución de los Indicadores de Empleo e Ingresos por Departamentos, 2007-2018 (INEI (ed.); Primera Ed). https://www.inei.gob.pe/media/MenuRecursivo/publicaciones_digitales/Est/Li b1678/libro.pdf

INEI. (2018). Características del Hogar. Perú: Perfil Sociodemográfico, 2017, 353-392. https://www.inei.gob.pe/media/MenuRecursivo/publicaciones_digitales/Est/Li b1539/cap06.pdf

INEI. (2020). INEI, Base de datos. https://www.inei.gob.pe/bases-de-datos/

Miranda, Ana; Alfredo, M. (2018). POLÍTICAS Y LEYES DE PRIMER EMPLEO EN AMÉRICA LATINA TENSIONES ENTRE INSERCIÓN Y CONSTRUCCIÓN DE TRAYECTORIAS. Revista de Ciencias Sociales, 31(42), 79-106.https://doi.org/10.26489/rvs.v30i41.4

Muñoz Martinez, D. (2018). El mercado de trabajo y las políticas de empleo juvenil: los casos de Chile y Uruguay (Issue July) [Universidad Comlutense de Madrid]. https://www.researchgate.net/profile/Daniel_Munoz_Martinez2/publication/33 0258268_El_mercado_de_trabajo_y_las_politicas_de_empleo_juvenil_los_casos_ de_Chile_y_Uruguay/links/5d386654a6fdcc370a5bf315/El-mercado-de-trabajoy-las-politicas-de-empleo-juvenil-1

Naciones Unidas/CEPAL. (2019). La Agenda 2030 y los Objetivos de Desarrollo Sostenible: una oportunidad para América Latina y el Caribe. Objetivos, metas e indicadores mundiales. In Publicación de las Naciones Unidas. https://repositorio.cepal.org/bitstream/handle/11362/40155/24/S1801141_es.p df 
Olmos, A. (2011). Jóvenes Bicentenario: Reflexiones a partir de las políticas públicas en vulnerabilidad, empleabilidad y juventud. Actuel Marx/ Intervenciones $\mathrm{N}^{\circ}, 10$, 193-210. http://www.actuelmarxint.cl/sitio_2017/ wp-content/uploads/2018/ 10/10.-Olmos-A.-Jóvenes-bicentenario-reflexiones-a-partir-de-las-políticas-públi cas-en-vulnerabilidad-empleabilidad-y-juventud. pdf

Pérez Aguilar, D. (2019). Jóvenes y desempleo: Desafío a las políticas públicas. Experiencia de Programa Entra21 en Chile. Journal of Chemical Information and Modeling, 53(9), 1689-1699. https://repositorio.unicartagena.edu.co/bitstream/ handle/11227/7289/Yolibeth Buelvas - Sistematizacion de experiencias 2018. pdf?sequence $=1$

Pérez Espíritu, L. (2020). PROYECTO DE LEY QUE ESTABLECE LA CUOTA DE EMPLEO JUVENIL (p. 142). https://leyes.congreso.gob.pe/Documentos/ 2016_2021/Proyectos_de_Ley_y_de_Resoluciones_Legislativas/PL05280202005 19.pdf

Quitral Rojas, M. (2012). Crisis y Bicentenario. Algunas consideraciones económicosociales. Revista Latinoamericana de Desarrollo Económico, 18, 177-199. https://doi.org/10.35319/lajed.201218135

Salvia, A. (2013). Juventudes, problemas de empleo y riesgos de exclusión social: El actual escenario de crisis mundial en la Argentina. Biblioteca Digital Fundación Friedrich Ebert, 17. http://library.fes.de/pdf-files/iez/09698.pdf

Tarazona Leyva, Gloria Melina; Moscoso Paucarchuco, Ketty Marilú; Casas Cornelio, Cristian Jordy; Peña Chinguel, Reysenia; Quispe Santos, Claudia Adelayda; Sillo Campos, Anali Karin; Palomino Ramirez, K. J. (2021). Youth work resilience in times of pandemic (F. E. de la U. Roosevelt (ed.); Primera Ed). http://revistas.uroosevelt.edu.pe/index.php/VISCT/article/view/79/130

Thezá, M. (2010). Políticas públicas de Juventud: tránsito y desafíos para el bicentenario Instituto Nacional de la Juventud (p. 6). https://d1wqtxts1xzle7.cloudfront. net/30869694/Politicas_publicas.pdf?1362602775=\&response-content-dispositio $\mathrm{n}=$ inline $\% 3 \mathrm{~B}+$ filename $\% 3 \mathrm{DPolitica}$ _publica_de_juventud_transito_y.pdf\&Expir es $=1610146469 \&$ Signature $=J 6 E p k W t a g 7 N \sim N N W s O u I O-k t E d U c D p D j o h d U U$ $\mathrm{Y} \sim \operatorname{drzxBgBbmfHC}$ 